\title{
Public Health Ethics: Resource Allocation and the Ethics of Legitimacy
}

\section{Kristine Bærøe}

The Ethics Programme, University of Oslo, Norway

\section{Introduction}

Public health ethics is a relatively new academic field. Crucially, it is distinguished from traditional medical ethics by its focus on populations rather than individuals [1,2]. Still, the ethics of public health cannot be perceived completely detached from the ethics of individuals, as populations are made up of individuals. One issue that clearly falls within the intersection of a population- and an individualbased perspective on ethics is resource allocation. Resource allocation takes place at various stages within the organisation of healthcare, i.e. at the micro-, meso- and macro level [3]. Resources are almost always limited, with the consequence that some healthcare is prioritised while other care is rationed. In this manner, resource allocation creates winners and losers; those who get the best care available, those who do not receive the best care and those who do not receive care at all. It seems prudent to assume that any adequate public health ethics involved in population-based decision-making will have to address all ethical aspects of resource allocation, all the way from the macrolevel policy-making process to the micro-level implementation where it affects specific and identifiable individuals. More specifically, such an ethics must be able to deal with the ethical tensions arising between population-based concerns framing the policy design process, and individual concerns in the realisation of the resource allocations. In the following, I will identify an inherent ethical tension involved in 'legitimate resource allocation', which is related to the challenge of meeting the ethical requirements from a population- and an individualbased perspective at the same time.

\section{Clarifying assumptions}

Let us assume that the regulative idea that all people are to be treated as moral equals is valid in all health-related activity. This normative idea can serve as a unifying conceptual basis common to both an individual and population level of analysis. The idea already informs democratic constitutions, human rights, welfare services and the most broadly recognised ethical theories in the West [4]. It does not initially make any substantive, theoretical claim on how moral equality is to be brought about in general and in healthcare contexts in particular. Rather, it opens up for assessing the realisation of moral equality as a matter of contextualisation, both according to different perspectives (such as a population- and individual-based one) as well as within distinct perspectives. For these reasons, considering the preservation of moral equality as a constituting structure of what ethics requires of us in healthcare organisations in general and resource allocation in particular should not come as a provocative suggestion.

For the following argument, we also make a second presumption; we assume good health is of significant importance to people. Together, these two general assumptions help us relate the ethical analysis at the two levels. At the individual level of analysis, practical responses to individual health needs can be ethically assessed in terms of whether the efforts realise the individual patient as the moral equal to other patients or not. On a parallel track, at the population level of analysis, targeting a number of allocations-protective, preventive, curative or rehabilitating-aiming to improve the poor health of specified populations can be assessed in terms of realising the moral equality among populations. Additionally, for both levels go that exploitation, ignorance or indifference to exposed needs do not confirm moral equality between those in need and those with the means and powers to do something about it.

At the same time, realising moral equality does not necessarily mean that any kind of need is automatically ethically entitled to interventions. Roughly, ethical claims might be constrained by i) the absolute importance of the need, and ii) the relative importance of the need. The absolute importance of a need varies with how much is at stake for the patient(s) if not treated; the more beneficial an available intervention is, the less moral equality between the provider and patient/ population is realised if the provider can, but does not provide it. The relative importance of a need determines the importance of a need compared with the needs of others. Assessment of relative importance is typically called for when more than one person is entitled to the same resources and these are limited. When considering the relative importance of needs, moral equality among care receivers (individuals or populations) is confirmed by efforts to justify the selection one is forced to make; those whose care is rationed are not of less worth morally, rather their needs are for some specified reasons evaluated as less pressing.

From population considerations to individual impact: The ethics of resource allocation

I have sketched out a common general structure for ethical analysis at the population and individual level. A crucial question for public health ethics to address is then: Do ethically acceptable resource allocations that confirm moral equality at a population level of analysis also confirm moral equality of individuals affected by the decisions when implementing the policy at the individual level? We may be tempted to jump to the conclusion that any ethically acceptable results of population-based resource allocation also would be ethically acceptable when implemented among individuals. Unfortunately, the relation between the levels of analysis is more complicated than that It turns out we cannot expect the ethics of population-based decisionmaking to coincide with the ethics of individual healthcare provision.

Firstly, ethical conclusions about acceptability for individuals are subjected to argumentative restrictions different from those that apply to populations. At the population level of justification, considerations about ethically acceptable allocations of resources are subjected to methodologies that allow for consistent comparison among populations. This puts constraints on what can count as a relevant feature of ethical concern [5]. Relevance is restricted to the kinds of

Corresponding author: Kristine Bærøe, The Ethics Programme, University of Oslo, Norway, E-mail: kristine.baroe@ifikk.uio.no

Received October 29, 2011; Published November 06, 2011

Citation: Bærøe K (2011) Public Health Ethics: Resource Allocation and the Ethics of Legitimacy. J Clinic Res Bioeth S4:e001. doi:10.4172/2155-9627.S4-e001

Copyright: @ 2011 Bærøe K. This is an open-access article distributed under the terms of the Creative Commons Attribution License, which permits unrestricted use, distribution, and reproduction in any medium, provided the original author and source are credited. 
generic features that are shared by a critical number of people adding up to a population, whatever that number might be. Also, the relevance is restricted by standardised, measurable features that enable comparison among different populations (with summary measures such as HALY, DALYs). At the individual level, on the other side, relevant features to take into account when considering acceptable claims are not subjected to the same restrictions. Any concern that seems to matter in the sense that moral equality would be violated if that concern were not responded to may be relevant for judgments about what acceptable allocation requires. Also, what matters in this sense may differ from person to person quite independently of what objective features of the psychological or physiological conditions are addressed. Such concerns would, however, have to be filtered for 'reasonableness' somehow, in order to count as an 'objectively considered' relevant concern for anyone in a similar situation; for instance the way Scanlon suggests in his book, What We Owe to Each Other [6]. Individual cases are embedded in complex social settings. These allow for much richer characterisations of individuals, and provide a broader background for justification of relevant ethical concerns when the response to individual needs is considered. There are more ways to violate moral equality at this level than at the population level, where relevant features per definition are characteristics several patients have in common. At the individual level, we should then expect a broader scope of concerns with the potential of affecting the results of justified ethical requirements in the contexts of allocating scarce resources.

Secondly, decisions based on populational and individual concerns respectively also require different kinds of efforts to confirm that equal treatment is provided. At the population level, confirming moral equality in the process of allocating resources is realised when populations are adequately registered and accounted for according to the key set of data, which in turn is subjected to justified norms for assigning relative weight and enabling fair comparison. From a consequential point of view, public health policies create winners and losers when some are prioritised to targeted interventions and others are not. At the point of implementation, the way the non-prioritised individuals are followed up in terms of confirming moral equality is a matter of further ethical concern. Has it been explained to them why they do not get what they could have? Is their disapproval of the decision made known, and any reasons they may provide, registered? Are they offered any way to appeal the decision? Promoting moral equality at this level encompasses the support of human capacities necessary to live a good life in general such as attaining knowledge and understanding as well as engaging in reflection, communication and deliberation. If these capacities are being oppressed or neglecteddeliberatively or not-when people are affected by resource allocations, moral equality between decision-makers/providers and individual patients is not confirmed. Especially for severely ill people, there may be a lot at stake when being denied care with potential effect. Ignoring their need to understand the decision, as well as their views on the acceptability of the decision, violates moral equality. This is regardless of how well-justified the population-based resource allocation decision was in the first place.

It follows from these arguments that we cannot conclude that the 'spheres of ethical justification' involved in resource allocation necessarily coincide. Confirmation of moral equality at these different levels of ethical analysis may encounter different relevant concerns, as well as different calls for action. Therefore, we can neither conclude that ethically acceptable population-based allocation automatically transfers ethical acceptability to the individual level where allocations are implemented. Concerns that logically cannot be picked up and fed into the decision-making process at the population level may violate moral equality if not properly responded to at the point of implementation.

\section{Implications for legitimate resource allocations}

If we cannot presume coherence in ethical conclusions at the different levels of analysis involved in resource allocation, then how should ethically adequate justification of resource allocation be carried out? The authority of decision makers to make morally legitimate resource allocations (which must be distinguished from politically legitimate allocations) must be based on a decision-making process that is sensitive to the different levels of ethical justification involved.

Interestingly, allegedly legitimate resource allocations can be categorised according to the different stress put on the different levels of ethical analysis. On the one side, when emphasising populations, moral authority is conferred on experts trained to interpret and assess population data and in making justified normative trade-offs. On the other side, when emphasising individuals, moral authority is conferred on decision-making procedures, including the participation of non-expert stakeholders. In the first case, legitimacy is justified epistemologically, in terms of evidence-based and technically wellcalculated solutions and normatively justified weighting. In the other case, legitimacy can be justified i) politically, i.e. in terms of democratic, public participation and empowerment, ii) epitemologically, i.e. in terms of deliberation, drawing on relevant experience among stakeholders, and iii) educationally in terms of making the rationale(s) for the allocation comprehensible for those affected.

\section{Puzzle}

The following puzzle seems inherent in the ethics of legitimate resource allocation: When merely stressing the moral authority of experts, technically precise and normatively consistent-and in that sense well-justified allocations at the population level- assessment can be achieved. When stressing the involvement and consensus of non-expert stakeholders, consistent similar assessment is less likely to occur due to the non-expert's lack of adequate knowledge and training. Concerns expressed at the individual level might merge with population concerns, and the rigour of academic reasoning may be hard to keep on track. In such cases, according to the standards of population level, justification might not be reached. On the other hand, moral equality is realised at the individual level by turning affected people into active, empowered participants with the opportunity to voice their concerns from their particular points of view before the decision has been made. Also, this involvement supports stakeholders with the opportunity to understand the reasons that structure the resource allocations. In addition then, involvement also enables those affected to provide better-targeted criticism of the allocation when reasonable, but ignored concerns are raised at the point of implementation. I would like to underscore that this latter impact of allowing non-expert participation extends further than the aim of respecting the political autonomy of stakeholders-it may also contribute to epistemic empowerment of the people affected as well as potentially to the epistemic refinement of the decision-making process. For these reasons, the puzzle presented here is not equal to what has been labelled 'the democracy problem'. This problem is briefly summed up as the risk of producing morally arbitrary decisions by letting the democratic rule of majority overrule the search for the best justified solution on the one side, and the undemocratic process implied by opening up for judging issues of fairness based on an extra-democratic, moral principle, on the other $[7,8]$. 
So when organising ethical resource allocations, we seem forced to make a trade-off between two legitimate calls for realising moral equality, none of which are sufficient but both are necessary: consistent and well-justified expert approaches versus political participation and potentially valuable epistemological contributions and educational empowerment of stakeholders. To go for the first alternative may strengthen the ethical validity of the allocation decision within the sphere of population-based justification, at the price of making the rationale too complex and unavailable to grasp for those affected. The other alternative may strengthen the ethical responses called for at the point of implementing the allocation but at the price of renouncing the requirement of normative validity and epistemological stringency from a population-level point of view. Either way, confirmation of moral equality appears incomplete and may be linked with questionable decisions.

\section{Wriggling a way out of the puzzle}

A way out of this puzzle would be to organise legitimate resource allocations in a way that addresses the divergent calls for ethical justification. The influential framework, 'Accountability for Reasonableness' (A4R), can be seen to do exactly this [9]. This framework consists of four conditions to take into account when setting limits to healthcare. One of the conditions is that the rationale for resource allocations should be made public in order to enable justified criticism and revision. Another condition requires the relevance of the criteria to be fed into the procedure of decision-making to be assessed in terms of 'reasonableness'. This part of the framework reflects the ambition of producing a certain kind of normatively justified epistemological outcome of the process. A third condition is that those being affected must have the opportunity to appeal the decision, and the fourth condition is that there are either voluntary or public regulations of the priority-setting process that ensure the three previous conditions are met. The A4R framework clearly acknowledges that resource allocations are stretched out over the two levels of ethical analysis. It can be questioned though, whether more concern about enabling those carrying the burdens of the allocation decisions to understand the reasons they don't get what they could have had, should be better integrated within the framework. By doing so, the ethics at the individual level would be more adequately addressed. Also, this framework could have been strengthened if more reliable strategies to systematically pick up relevant concerns from those affected had been established. As it now stands, it is left to the patients and proxies with the surplus energy and resources to go through the process of appeal.

Another way of organising resource allocations that are sensitive to both the population and the individual level of ethical analysis can be found in the combination of 'multi-criteria decision-making analysis' (MCDA) and discrete choice experiments (DCE) among stakeholders [10-12]. The strategy of MCDA is to break down the complexity of concerns at a population level by identifying a comprehensive set of criteria and establishing the performance of interventions on those criteria in a so-called performance matrix. Moreover, this approach helps illuminate the implicit weighting and trade-offs taking place when reaching an allocation decision. By help of discrete choice experiments, the weight the decision-makers put on the different criteria can be used to rank the interventions accordingly.
Such a ranking list serves as a point of departure for further discussions about how to allocate the resources. The MCDA approach has the major advantage of making complex decision-making transparent. It thereby allows for targeted criticism. By breaking down complex decisions into easier understandable criteria, those affected by the allocation can also more easily be educated. Furthermore, this approach makes it possible to involve stakeholders both in deciding on relevant criteria and taking part in the weighting process carried out in terms of DCE. However, more research into how the combination of MCDA and DCE should be should be arranged to get the best justified epistemological outcome by the involvement of experts, politicians and different kinds of stakeholders is needed for this approach to adequately address both levels of ethical analysis.

\section{Conclusion}

The discrepancy between the distinct levels of ethical analyses involved in resource allocations produces an inherent ethical tension within the whole business of claiming legitimate decisions. An adequate public ethics needs to sort out how to strike the right balance when addressing both spheres of ethical justification, or alternatively, find a way to reconceptualise the entire constellation of concerns embedded in the two levels. This challenge remains an important task for public health ethics research to clarify. Next, it rests with governing authorities to realise ethical public health accordingly.

\section{References}

1. Kass N. E (2001) An Ethics Framework for Public Health. Am J Public Health 91: $1776-1782$.

2. Dawson A (2011) Resetting the parameters: Public health as the foundation for public health ethics, in Public Health Ethics: Key Concepts and Issues in Policy and Practice. Cambridge University Press.

3. Kapiriri L, Norheim OF, Martin DK (2007) Priority setting at the micro-, mesoand macro-levels in Canada, Norway and Uganda. Health Policy 82: 78-94.

4. Gosepath S. Equality (2007) The Stanford Encyclopedia of Philosophy, [cited 2011 7th April]

5. Bærøe K (2008) Priority setting in health care: On the relation between reasonable choices on the micro-level and the macro-level. Theor Med Bioeth 29: 87-102.

6. T.M. Scanlon (1998) What We Owe to Each Other. Cambridge, Mass: The Belknap Press of Harvard University Press.

7. Daniels N, Justice and justification: Reflective equilibrium in theory and practice Cambridge Studies in Philosophy and Public Policy, Cambridge [England] New York: Cambridge University Press. 317-326.

8. Fleck, L., Healthcare (2001) Justice and Rational Democratic Deliberation. The American Journal of Bioethics 1: 20-21.

9. Daniels N, Sabin J, (2002) Setting Limits Fairly. Can We Learn to Share Medical Resources? Oxford: Oxford University Press.

10. Baltussen R, Niessen L (2006) Priority setting of health interventions: The need for multi-criteria decision analysis. Cost Effectiveness and Resource Allocation 4: 14

11. Baltussen R, Youngkong S, Paolucci F (2010) Multi-criteria decision analysis to prioritize health interventions: Capitalizing on first experiences. Health Policy 96: 262-264

12. Youngkong $S$, Baltussen $R$, Tantivess $S$, Koolman $X$, Teerawattananon $Y$ (2010) Criteria for priority setting of HIVIAIDS interventions in Thailand: a discrete choice experiment. BMC Health Serv Res 10: 197 